\title{
Software educativo CHAKANA: una propuesta para desarrollar la inteligencia lógico-matemática de estudiantes universitarios
}

\section{Educational software CHAKANA: a proposal to develop logical-mathematical intelligence for college students}

\author{
Guido Mazón Fierro ${ }^{1}$ \\ ${ }^{1}$ Universidad Nacional de Chimborazo - UNACH, Riobamba, Equador
}

Correo a/Mail to: Guido Mazón Fierro gmazon@unach.edu.ec

Financiamiento/Funding: Universidad Nacional de Chimborazo, UNACH

Recibido/Submitted: 27 Oct. 2015; Aceptado/Approved: 10 Nov. 2015

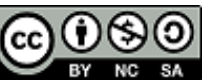

\begin{abstract}
Copyright (c) 2015 Mazón Fierro, G.. Todo el contenido de la revista (incluyendo instrucciones, política editorial y modelos) está bajo una licencia Creative Commons Atribución-NoComercial-Compartirlgual 3.0 No Adaptada. Siendo publicados por esta revista, artículos son de libre uso en ambientes educativos, de investigación y no comerciales, con atribución de autoría obligatoria. Más información en
\end{abstract} http://ojs.c3sl.ufpr.br/ojs2/index.php/atoz/about/submissions\#copyrightNotice.

\begin{abstract}
Resumen
Introducción: al evidenciar que los jóvenes estudiantes del segundo semestre de la Carrera de Ingeniería en Sistemas y Computación de la Universidad Nacional de Chimborazo (Ecuador) poseen un nivel básico de lógica deductiva, y les cuesta resolver problemas de razonamiento con agilidad - lo cual repercute en un bajo rendimiento en la materia de matemática - el objetivo del presente estudio es facilitar un recurso didáctico asistido por computador para desarrollar la inteligencia lógico-matemática, como es el software educativo Chakana.

Método: Cincuenta estudiantes compusieron el universo de investigación y una muestra aleatoria de 19 fue envolucrada en los testes. En la primera fase se diseñó y se creo el Software Educativo Chakana con un enfoque en los problemas evidenciados y, en la segunda fase - para la aplicación del software - se comenzó valorando un test inicial sobre la inteligencia lógico-matemática para luego de 14 semanas trabajar con el software Chakana, y posteriormente aplicar el test final.

Resultados: la investigación demuestró cómo la elaboración y aplicación del software de razonamiento lógico Chakana tiene un impacto favorable en el desarrollo de la inteligencia lógico-matemática de los estudiantes, en especial el análisis de relaciones lógicas y de analogías. Conclusión: de manera general los estudiantes manejaran la herramienta y la encontraron actractiva. Se ha observando su aceptación además del desarrollo de la inteligencia lógico-matemática que se vio reflejado en las notas de la asignatura de matemática.
\end{abstract}

Palabras clave: Software Educativo; Metodología THALES; Inteligencias Múltiples; Razonamiento lógico-matemático

\begin{abstract}
Introduction: Young students of the second semester of the School of Systems and Computer Engineering of the National University of Chimborazo (Ecuador) have a basic level of deductive logic. Therefore they show dificulties to solve reasoning problems, which leads to a poor performance in the field of mathematics. Due to this previous analysis, the objective of the study was to provide a computer-assisted teaching resource to help the developing of logical-mathematical intelligence.

Method: 50 students were identified as the study universe and 19 students were enrroled - as an aleatory sample - for the tests. The first test phase (of two) included the design - based on the problems identified - and the creation of the Chakana Educational Software. Along to the software implementation, a pretest on logical-mathematical issues was applied being used as the baseline for the study. Fourteen weeks later the second phase begun with the use of the Chakana sofware, and a final test was applied.

Results: It was showed - by using the Chakana software - the students improved their logical-mathematical reasoning and were more capable to face analogy challenges.

Conclusion: The students handled this digital tool that proved to be atractive and helpfull as an aid to their logical-mathematical skills; which was seen through the better grades at the subject.
\end{abstract}

Keywords: Educational software; THALES Methodology; Multiple Intelligences; Logical-Mathematical reasoning

\section{INTRODUCCIÓN}

En los actuales momentos en los cuales el mundo se desarrolla, no se podría entender una sociedad moderna sin la ayuda de las ciencias informáticas o de un computador, y es así que el uso de programas computacionales o diferentes tipos de software han influenciado a todas las áreas del conocimiento (Rodríguez, 2013). Por otra parte, la Teoría de las Inteligencias Múltiples (IM), estudiada por el profesor estadounidense Howard Gardner, ha dado un nuevo enfoque a la manera de percibir la inteligencia. Gardner (2013) la define como una capacidad que no es innata e inamovible, como muchas veces se piensa, sino que puede ser desarrollarla.

Es así que se va a citar el trabajo de Suárez, Maiz, y Meza (2010) en donde se destaca que, el concepto de inteligencia ha cambiado de manera expedita ya que esta se percibía como estática, innata e influenciada por la herencia y cultura, deduciéndose así que cada persona posee diferentes potenciales cognitivos. Así, en el ámbito educativo, la teoría del profesor Gardner proporciona información relevante sobre estilos de aprendizaje, contribuyendo a percibir a los estudiantes como entidades que aprenden de maneras diferentes, lo 
que debiera generar estrategias metodológicas diversas para un mismo contenido, potenciando en el estudiante la posibilidad de reconocer sus capacidades cognitivas al máximo (Suárez et al., 2010). En este trabajo se obtuvo importante información con respecto a cómo un recurso innovador didáctico puede generar cambios significativos en el el proceso de enseñanza-aprendizaje de los educandos.

En vista de que los docentes en su continua actividad profesional se encuentran en el aula de clases con grupos heterogéneos y que presentan diferentes niveles en el desarrollo de sus capacidades cognitivas, una de las falencias más frecuentes en materias de ciencias exactas es que a los estudiantes les cuesta plantear y resolver problemas de razonamiento con agilidad. Se verifica que tienen un nivel básico de lógica deductiva o no suelen realizar analogías de manera acertada. Es por esto que la presente propuesta del trabajo investigativo enlaza dos aristas, la inteligencia y la utilización de las tecnologías computacionales a través de un software.

Como menciona Cataldi, Lage, Pessacq, y García-Martínez (2013), se debe utilizar una mitología extendida para la creación de software educativo desde una visión integradora en donde existan elementos para educar y fortalecer en la inteligencia lógico-matemática en los estudiantes. Existen dos áreas principales - o campos específicos - de la inteligencia lógico-matemática, que se pueden desarrollar en los estudiantes con mayor predisposición, como es el pensamiento matemático y la lógica deductiva (Campbell, Campbell, \& Dickinson, 2010).

Así pues, el objetivo del presente estudio es facilitar un recurso didáctico asistido por computador llamado Software Educativo Chakana para desarrollar la inteligencia lógico-matemática en estudiantes universitarios. La hipótesis a comprobar es si la elaboración y aplicación del software de razonamiento lógico Chakana tiene impacto en el desarrollo de la inteligencia lógico-matemática de los estudiantes en el ámbito del pensamiento matemático, en el área de la lógica deductiva, mediante la utilización de análisis de relaciones lógicas y de capacidad de efectuar analogías.

\section{PROCEDIMIENTOS METODOLÓGICOS Y RESULTADOS}

La investigación posee un diseño experimental, y es de tipo analítico, prospectivo de carácter longitudinal de campo. El método científico utilizado es inductivo-deductivo a través de sus diferentes fases.

Se ha elaborado un software educativo utilizando y respetando rigurosamente las seis fases de la metodología THALES propuesto por (Madueño \& Ruiz, 2002), el cual posee como principal característica el ser un modelo híbrido no lineal. En la Figura 1 se ilustran las etapas desarrolladas.

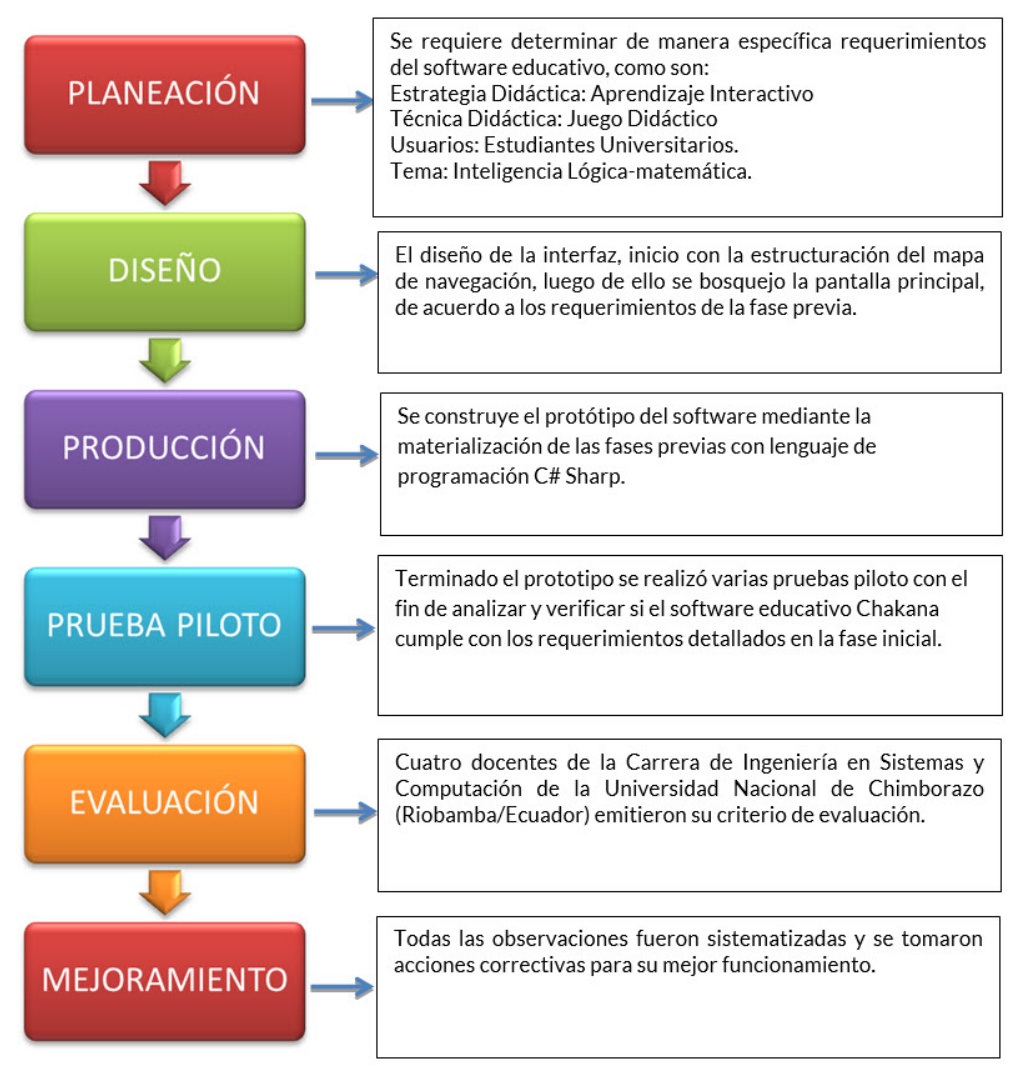

Figura 1. Etapas Metodológicas de la elaboración del Software.

Fonte: El autor. 
Una vez terminada la fase de elaboración se planteó una recolección de datos de naturaleza cuali-cuantitativa. Se desarrolló, junto a los estudiantes, la fase de aplicación del Software Educativo Chakana en 16 semanas así divididas:

a) la primera semana se efectuó el test inicial en la cual se aplicó un cuestionario de test inicial de (Castaño, 2014) de donde se obtuvo la línea base o diagnóstico de la situación de la muestra;

b) de la semana dos a la quince se realizó el trabajo con el Software Educativo Chakana y la guía didáctica elaborada para el estudiante (durante cinco días a la semana por un lapso de 15 minutos), . En esta etapa se anotaron en las fichas de registro los resultados del trabajo con el software. Tales fichas evidencian una bitácora de lo que va aconteciendo en el transcurso de la investigación;

c) la semana 16 terminó con un test final de (Castaño, 2014) que permitió evidenciar una evaluación terminal al grupo de estudiantes de la muestra y su comparación con el test inicial.

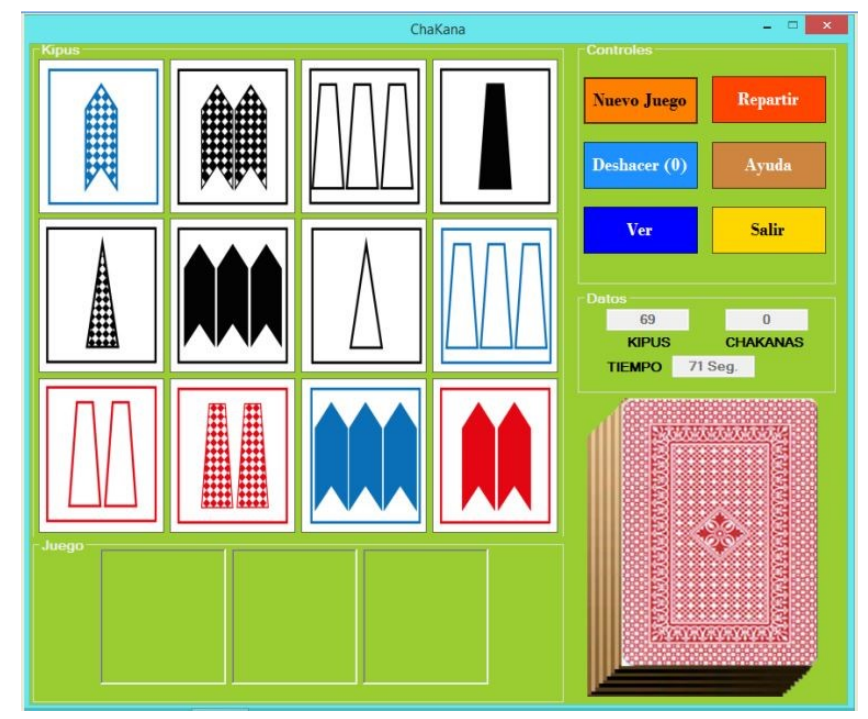

Figura 2. Ventana Principal del Software Educativo Chakana.

Fonte: El autor.

En la etapa de aplicación se trabajó con los estudiantes dos aspectos de la inteligencia lógico-matemática: a) el pensamiento matemático a través del análisis de relaciones lógicas; y b) el área de la lógica deductiva, realizando analogías de esquemas gráficos como, por ejemplo, la figura que se muestra a continuación que detalla como el usuario del Software educativo construye una Chakana. Para tal, el usuario tiene que escoger tres fichas y, para estas, relacionar lógicamente cuatro variables como son: cantidad, relleno, color y figura (se realizan relaciones de igualdad y diferencia entre cada ficha para formar una Chakana) (Figura 3).

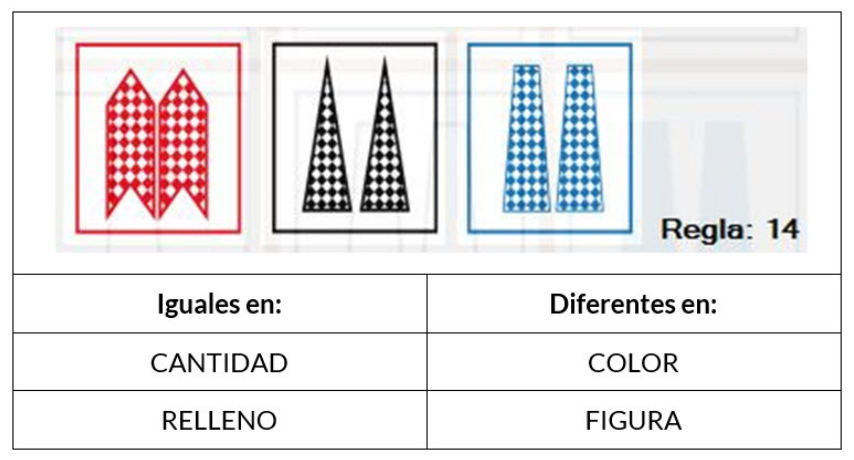

Figura 3. Formación de una Chakana.

Fonte: El autor.

Existió un universo de 50 estudiantes del segundo semestre de la Escuela de Ingeniería en Sistemas y Computación de la Universidad Nacional de Chimborazo (Riobamba/Ecuador) en el período académico Septiembre 2013 - Febrero 2014. Para el tamaño de la muestra se aplicó un muestreo aleatorio simple probabilístico, es decir, para encontrar una muestra representativa con relación a la población se consideró la fórmula para poblaciones finitas no numerosas dando como resultado 19 estudiantes como grupo de análisis.

Para evidenciar la veracidad de la hipótesis - la cual menciona que la elaboración y aplicación del software de razonamiento lógico tiene un impacto favorable en el desarrollo de la inteligencia lógica matemática en los estudiantes (mediante el análisis de relaciones lógicas y capacidad de efectuar analogías) - se empleó la 
teoría de la significancia estadística (Supo, 2010), en el cual se aplicó un nivel de confianza del 95\% (es decir, una significancia $\alpha=0,05$ ). De acuerdo al nivel de la investigación, la naturaleza de las variables y tipo de investigación se puedo seleccionar que la prueba estadística adecuada es $t$ de student para muestras relacionadas, con la lectura del p-valor que es el $t$ de student calculado $\left(t_{c}\right)$. Mediante la utilización de la hoja de cálculo Microsoft Excel y el valor de significancia $\alpha=0,05$, se rechazaron las hipótesis especificas nulas $H_{0}$ y se aceptaron las hipótesis especificas alternativas $H_{1}$.

Los estudiantes antes de la aplicación del software de razonamiento lógico Chakana no adiestraban el análisis de relaciones lógicas ni realizaban analogías de esquemas gráficos. Luego de haberse aplicado el software Chakana durante cinco días a la semana por un lapso de 15 minutos, los estudiantes han pasado de un nivel promedio de $52 \%$ a un $67 \%$ (Figura 4) lo que muestra que existe una progresión porcentual en el tipo de inteligencia lógicomatemática con respecto al pensamiento matemático y razonamiento deductivo. Puesto que el test inicial y el test final cuantificaban estas dos variables, se puede afirmar que el software contribuyo en el desarrollo de sus capacidades cognitivas, tales como memoria, resolución de problemas, comprensión, solución de esquemas lógicos y establecimiento de analogías.

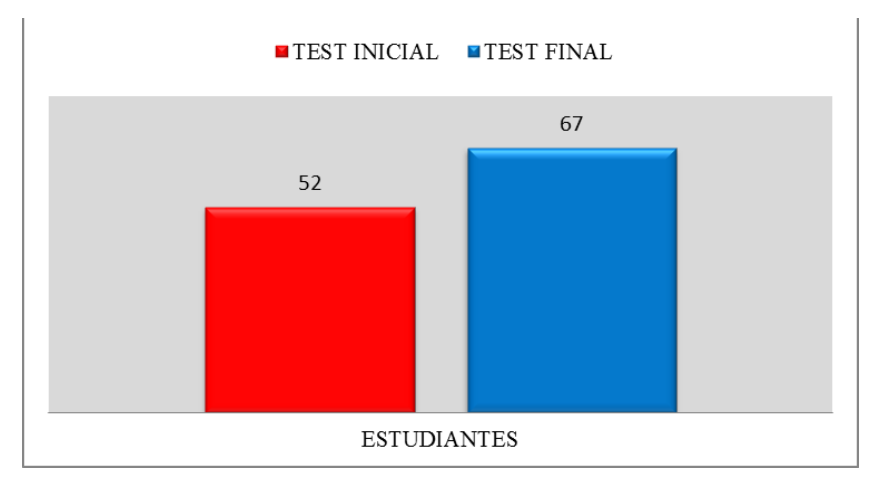

Figura 4. Promedio general test inicial y final de inteligencia lógico-matemática. Fonte: El autor.

Se evidencia en el incremento de la media aritmética del test inicial al test final lo que permite enunciar que el desarrollo del Software Educativo Chakana se constituye en una herramienta de refuerzo para desarrollar la inteligencia lógica-matemática en dos grandes áreas: el pensamiento matemático y la lógica deductiva.

\section{CONCLUSIONES}

Se facilitó un recurso didáctico asistido por computador como es el Software Educativo Chakana - con su respectiva guía didáctica para uso del estudiante - que genera un impacto positivo y favorable en el desarrollo de la inteligencia lógico-matemática. En la muestra definida de estudiantes se pudo verificar que también se ha presentado una innovación educativa a través de recursos didácticos digitales o entornos virtuales de aprendizaje interactivos, los cuales buscan mejorar el proceso enseñanza-aprendizaje en el aula de clase y en el ámbito académico en general.

Además, se pudo verificar que en la elaboración del software siguiendo la metodología THALES presenta grandes facilidades para enfocar y solucionar problemas educativos, siempre y cuando se cumpla cuidadosamente con cada una de sus fases que son factores determinantes en la construcción del programa y que garantizan la calidad del producto final.

Para la aplicación del Software Educativo Chakana, los estudiantes deben tomar muy en cuenta la guía didáctica en donde se encuentran elementos como requerimientos y procedimiento de instalación, además del guía del usuario, en lo referente a la parte técnica, para evitar inconvenientes y poder trabajar metodológicamente. Se incluyó en el experimento, lecturas que permiten hacer la inducción sobre la teoría de las inteligencias múltiples.

Es recomendable que los estudiantes colaboren positivamente en responder a los test inicial y final en los tiempos estipulados, de la misma forma respetar el tiempo asignado de utilización del Software Educativo Chakana.

Se sugiere realizar la implementación de nuevas versiones, con fines de fortalecer otras inteligencias múltiples y desarrollar nuevas aplicaciones en dispositivos móviles y, finalmente, se puede recomendar aplicar esta herramienta asistida por computador a estudiantes universitarios que muestren falta de adiestramiento en la inteligencia lógica-matemática. 


\section{REFERENCIAS}

Campbell, L., Campbell, B., \& Dickinson, D. (2010). Inteligencias múltiples usos prácticos para la enseñanza y aprendizaje. Buenos Aires: Troquel.

Castaño, O. (2014, 17 de oct.). Mentesenblanco. Descargado de http://www.mentesenblanco-razonamientoabstracto .com/razonamiento.html

Cataldi, Z., Lage, F., Pessacq, R., \& García-Martínez, R. (2013). Metodología extendida para la creación de software educativo desde una visión integradora. Revista Latinoamericana de Tecnología Educativa, 2(1), 59-64. Descargado de http://relatec.unex.es/article/view/11

Gardner, H. (2013). Estructuras de la mente: La teoría de las inteligencias múltiples. México D.F.: Basic Books.

Madueño, L., \& Ruiz, M. (2002). La organización del proyecto thales: una propuesta de informática educativa. Revista Latinoamericana de Tecnología Educativa, 1(2), 37-44. Descargado de http://relatec.unex.es/article/view/9

Rodríguez, J. (2013). Aprendizaje y educación en la sociedad. Barcelona: Sociedad Digital.

Supo, J. (2010, 20 de mar.). Bioestadistico.com. Descargado 12 mayo 2015, de https://www.youtube.com/watch?v= jq8C48KkdCA

Suárez, J., Maiz, F., \& Meza, M. (2010, abr.). Inteligencias múltiples: Una innovación pedagógica para potenciar el proceso enseñanza aprendizaje. Investigación y Postgrado, 25(1), 81-94. Descargado de http://www.scielo.org.ve/scielo.php ?pid=S1316-00872010000100005 \&script=sci_arttext

Cómo citar este shortpaper (APA):

Mazón Fierro, G. (2015). Software educativo CHAKANA: una propuesta para desarrollar la inteligencia lógicomatemática de estudiantes universitarios. AtoZ: novas práticas em informação e conhecimento, 4(2), 103 107. Descargado de: http://dx.doi.org/10.5380/atoz .v4i2.43691 